\title{
The beta exponentiated Lindley distribution
}

\author{
J. A. Rodrigues \\ Departamento de Ensino, Instituto Federal da Bahia \\ Feira de Santana, 44.096-486, Brasil \\ jailsondearaujo@yahoo.com.br \\ A. P. C. M. Silva \\ Departamento de Engenharia de Alimentos, Universidade Federal de São João Del Rei \\ Sete Lagoas, 35701-970, Brasil \\ apcmadeira.@hotmail.com \\ G.G. Hamedani \\ Department of Mathematics, Statistics and Computer Science \\ Marquette University \\ Milwaukee, Wisconsin 53201-1881 \\ Received 15 June 2014 \\ Accepted 16 December 2014
}

\begin{abstract}
In this work, we study the beta exponentiated Lindley distribution which includes as special cases several models such as the Lindley, exponentiated Lindley and beta Lindley distributions. Some structural properties of the proposed distribution are studied including expressions for the moments. The estimation of parameters using the methods of moments and maximum likelihood is also discussed. The flexibility of this distribution is illustrated in an application to a real data set.

Keywords: Akaike information criterion; beta distribution; Lindley distribution; maximum likelihood estimation; moments.
\end{abstract}

\section{Introduction}

In recent years, generalized distributions have been widely studied in statistics as they possess flexibility in applications. This is justified because the traditional distributions often do not provide good fit in relation to the real data set studied. For example, [1] proposed the beta-Pareto distribution and discussed its various properties. [2] introduced and studied the gamma-Pareto distribution. [3] proposed the Weibull-Pareto distribution. [5] studied the beta-Dagum distribution. [12] presented the beta-Weibull distribution. [15] defined a five-parameter beta Burr XII distribution and discussed its various properties. [16] introduced the Kumaraswamy generalized gamma distribution. [17] studied the gamma-exponentiated Weibull distribution and [18] studied the beta modified Weibull distribution. 
The Lindley distribution [11] is important for studying stress-strength reliability modeling. The cumulative distribution function $(\mathrm{CDF})$ of the Lindley distribution is given by:

$$
W(x)=1-\frac{\theta+1+\theta x}{\theta+1} e^{-\theta x}
$$

where $x>0$ and $\theta>0$ is a scale parameter. Some authors have proposed new distributions based on modifications of the Lindley model. [20] obtained the two-parameter Lindley distribution and discussed its various properties and applications. [19] obtained the negative binomial-Lindley distribution by combining the negative binomial and Lindley distributions. The three-parameter Lindley distribution was introduced by [6], who used the distribution for modeling survival data. An important extension/generalization of the Lindley distribution is the exponentiated Lindley distribution introduced by [14] with CDF:

$$
G(x)=W^{\alpha}(x)=\left(1-\frac{\theta+1+\theta x}{\theta+1} e^{-\theta x}\right)^{\alpha}
$$

where $\alpha>0$ is a shape parameter. The corresponding probability density function (PDF) is:

$$
g(x)=\frac{\alpha \theta^{2} e^{-\theta x}}{\theta+1}(1+x)\left(1-\frac{\theta+1+\theta x}{\theta+1} e^{-\theta x}\right)^{\alpha-1}
$$

In this article, we propose a new distribution that extend the exponentiated Lindley distribution. Some of the main structural properties of this distribution are derived. The flexibility of this distribution is illustrated in an application to a real data set.

The article is organized as follows. In Section 2 is defined the beta exponentiated Lindley distribution and some special sub-models are discussed. The failure rate function, moments, moment generating function and characteristic function are derived in Section 3. Characterizations of the new model are presented in Section 4. The estimation of the parameters using the methods of moments and maximum likelihood is discussed in Section 5. Finally, in Section 6 an application on a real data set is reported.

\section{The model}

Let $G(x)$ be the CDF of any random variable $X$. The CDF of a generalized class of distributions defined by [7] is given by

$$
F(x)=\frac{1}{B(a, b)} \int_{0}^{G(x)} t^{a-1}(1-t)^{b-1} d t
$$

where $a>0, b>0$ and $B(\cdot, \cdot)$ is the beta function defined by

$$
B(a, b)=\int_{0}^{1} t^{a-1}(1-t)^{b-1} d t
$$

. The corresponding PDF for (2.1) is given by

$$
f(x)=\frac{1}{B(a, b)} g(x)[G(x)]^{a-1}[1-G(x)]^{b-1}
$$

Replacing (1.2) in (2.1), we obtain a new distribution, called beta exponentiated Lindley (BEL), with CDF given by 


$$
F(x)=\frac{1}{B(a, b)} \int_{0}^{\left(1-\frac{\theta+1+\theta x}{\theta+1} e^{-\theta x}\right)^{\alpha}} t^{a-1}(1-t)^{b-1} d t
$$

The PDF corresponding for $F(x)$ is

$$
f(x)=\frac{\alpha \theta^{2}(1+x) e^{-\theta x}}{(\theta+1) B(a, b)}\left[1-\left(1-\frac{\theta+1+\theta x}{\theta+1} e^{-\theta x}\right)^{\alpha}\right]^{b-1}\left(1-\frac{\theta+1+\theta x}{\theta+1} e^{-\theta x}\right)^{\alpha a-1}
$$

Figure 1 shows the different graphs of PDF of BEL distribution for various values of the parameters $a, b, \alpha$ and $\theta$.
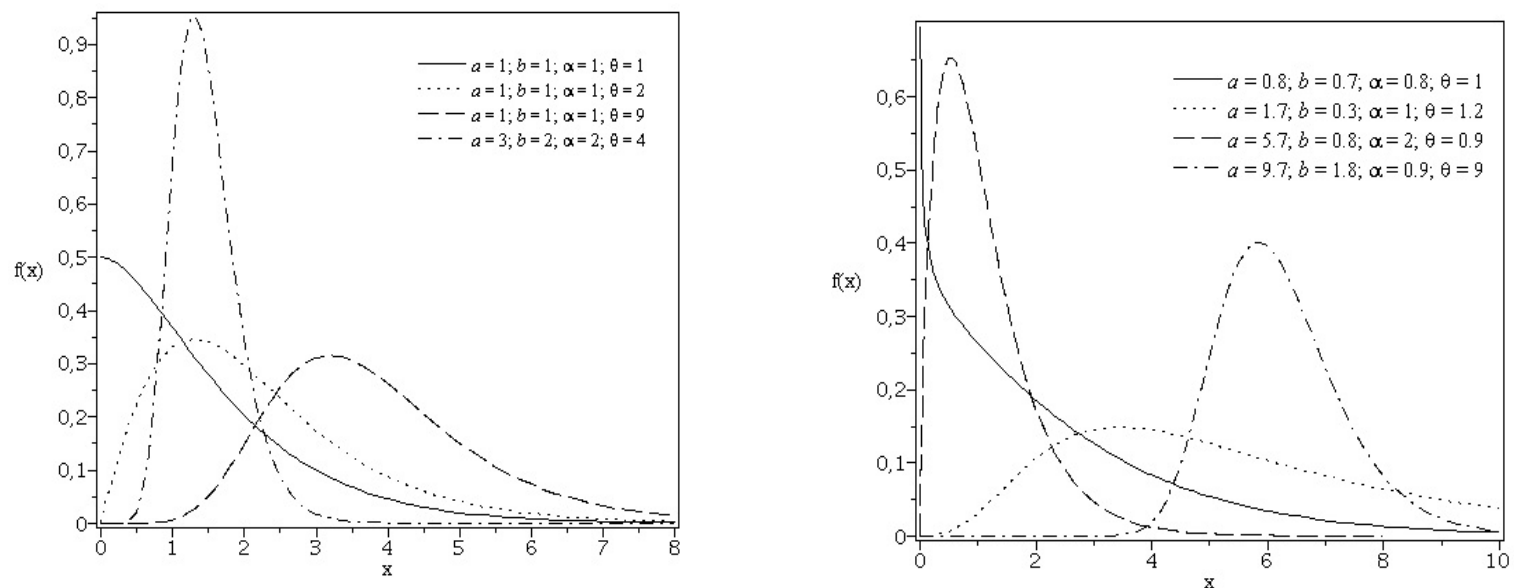

Fig. 1. The PDF of the BEL distribution for different values of the parameters.

\subsection{Submodels}

The following distributions are special of the BEL distribution:

(1) When $\alpha=1$, the BEL distribution is the beta Lindley (BL) distribution (see [13]), with the density given by:

$$
f(x)=\frac{\theta^{2}(1+x) e^{-\theta b x}}{(\theta+1) B(a, b)}\left(\frac{\theta+1+\theta x}{\theta+1}\right)^{b-1}\left(1-\frac{\theta+1+\theta x}{\theta+1} e^{-\theta x}\right)^{a-1} ;
$$

(2) If $b=1$, we have the exponentiated Lindley (EL) with parameters $\theta>0$ and $\alpha a>0$. The $\mathrm{PDF}$ is

$$
f(x)=\frac{\alpha a \theta^{2}(1+x) e^{-\theta x}}{(\theta+1)}\left(1-\frac{\theta+1+\theta x}{\theta+1} e^{-\theta x}\right)^{\alpha a-1} ;
$$

(3) If $a=b=1$, we have the EL with the PDF,

$$
f(x)=\frac{\alpha \theta^{2} e^{-\theta x}}{\theta+1}(1+x)\left(1-\frac{\theta+1+\theta x}{\theta+1} e^{-\theta x}\right)^{\alpha-1}
$$


(4) If $\alpha=b=1$, we have another EL distribution with parameters $\theta>0$ and $a>0$. The PDF is

$$
f(x)=\frac{a \theta^{2} e^{-\theta x}}{\theta+1}(1+x)\left(1-\frac{\theta+1+\theta x}{\theta+1} e^{-\theta x}\right)^{a-1} ;
$$

(5) If $a=b=\alpha=1$, we have the Lindley distribution with the PDF,

$$
f(x)=\frac{\theta^{2}}{\theta+1}(1+x) e^{-\theta x}
$$

\section{Properties of the model}

In the Section, we discuss some of the main properties of the BEL distribution.

\subsection{Expansions for the cumulative and density functions}

Theorem 3.1. The CDF of BEL distribution, for $\alpha(a+j)$ and $b$ real non-integers, is given by

$$
F(x)=\frac{\alpha}{B(a, b)} \sum_{j, k=0}^{\infty} \sum_{l=0}^{k}\left(\frac{\theta}{\theta+1}\right)^{l} \frac{(-1)^{j+k} \Gamma(b) \Gamma[\alpha(a+j)] x^{l} e^{-\theta k x}}{\Gamma(b-j) \Gamma[\alpha(a+j)+1-k](k-l) ! j ! l !}
$$

where $\Gamma(\cdot)$ is the gamma function defined by

$$
\Gamma(\alpha)=\int_{0}^{\infty} t^{\alpha-1} e^{-t} d t
$$

If $b$ is an integer, the index $j$ stops at $b-1$. If $\alpha(a+j)$ is an integer, the index $k$ stops at $\alpha(a+j)$.

Proof. The CDF for the BL distribution is given by

$$
\begin{aligned}
& F(x)=\frac{1}{B(a, b)} \int_{0}^{\left(1-\frac{\theta+1+\theta x}{\theta+1} e^{-\theta x}\right)^{\alpha}} t^{a-1}(1-t)^{b-1} d t \\
& =\frac{1}{B(a, b)} \sum_{j=0}^{\infty} \frac{(-1)^{j} \Gamma(b)}{\Gamma(b-j) j !} \int_{0}^{\left(1-\frac{\theta+1+\theta x}{\theta+1} e^{-\theta x}\right)^{\alpha}} t^{a+j-1} d t \\
& =\frac{1}{B(a, b)} \sum_{j=0}^{\infty} \frac{(-1)^{j} \Gamma(b)}{(a+j) \Gamma(b-j) j !}\left(1-\frac{\theta+1+\theta x}{\theta+1} e^{-\theta x}\right)^{\alpha(a+j)} \\
& =\frac{\alpha}{B(a, b)} \sum_{j=0}^{\infty} \frac{(-1)^{j} \Gamma(b)}{\Gamma(b-j) j !}\left\{\sum_{k=0}^{\infty} \frac{(-1)^{k} \Gamma[\alpha(a+j)]}{\Gamma[\alpha(a+j)+1-k] k !}\left(\frac{\theta+1+\theta x}{\theta+1} e^{-\theta x}\right)^{k}\right\} \\
& =\frac{\alpha}{B(a, b)} \sum_{j, k=0}^{\infty} \frac{(-1)^{j+k} \Gamma(b) \Gamma[\alpha(a+j)]}{\Gamma(b-j) \Gamma[\alpha(a+j)+1-k] j ! k !}\left(\frac{\theta+1+\theta x}{\theta+1}\right)^{k} e^{-\theta k x} \\
& =\frac{\alpha}{B(a, b)} \sum_{j, k=0}^{\infty} \sum_{l=0}^{k}\left(\frac{\theta}{\theta+1}\right)^{l} \frac{(-1)^{j+k} \Gamma(b) \Gamma[\alpha(a+j)] x^{l} e^{-\theta k x}}{\Gamma(b-j) \Gamma[\alpha(a+j)+1-k](k-l) ! j ! l !}
\end{aligned}
$$


Theorem 3.2. The PDF of BEL distribution, for $b$ and $\alpha(a+j)$ real non-integers, is given by

$$
f(x)=\frac{\alpha \theta^{2}(1+x)}{(\theta+1) B(a, b)} \sum_{j, k=0}^{\infty} \sum_{l=0}^{k}\left(\frac{\theta}{\theta+1}\right)^{l} \frac{(-1)^{j+k} \Gamma(b) \Gamma[\alpha(a+j)] x^{l} e^{-\theta(k+1) x}}{\Gamma(b-j) \Gamma[\alpha(a+j)-k](k-l) ! j ! l !}
$$

If $b$ is an integer, the index $j$ stops at $b-1$. If $\alpha(a+j)$ is an integer, the index $k$ stops at $\alpha(a+j)-1$.

Proof. The PDF for the BEL distribution is given by

$$
f(x)=\frac{\alpha \theta^{2}(1+x) e^{-\theta x}}{(\theta+1) B(a, b)}\left[1-\left(1-\frac{\theta+1+\theta x}{\theta+1} e^{-\theta x}\right)^{\alpha}\right]^{b-1}\left(1-\frac{\theta+1+\theta x}{\theta+1} e^{-\theta x}\right)^{\alpha a-1}
$$

For $a>0$ real non-integer and $|z|<1$, we have the power series

$$
(1-z)^{a-1}=\sum_{j=0}^{\infty} \frac{(-1)^{j} \Gamma(a) z^{j}}{\Gamma(a-j) j !}
$$

Using the series representation (3.6) in Equation (3.5), we can write

$$
\begin{aligned}
f(x) & =\frac{\alpha \theta^{2}(1+x)}{(\theta+1) B(a, b)} \sum_{j, k=0}^{\infty} \frac{(-1)^{j+k} \Gamma(b) \Gamma[\alpha(a+j)] e^{-\theta(k+1) x}}{\Gamma(b-j) \Gamma[\alpha(a+j)-k] j ! k !}\left(\frac{\theta+1+\theta x}{\theta+1}\right)^{k} \\
& =\frac{\alpha \theta^{2}(1+x)}{(\theta+1) B(a, b)} \sum_{j, k=0}^{\infty} \sum_{l=0}^{k}\left(\frac{\theta}{\theta+1}\right)^{l} \frac{(-1)^{j+k} \Gamma(b) \Gamma[\alpha(a+j)] x^{l} e^{-\theta(k+1) x}}{\Gamma(b-j) \Gamma[\alpha(a+j)-k](k-l) ! j ! l !}
\end{aligned}
$$

\subsection{Hazard rate function}

For a continuous distribution with $\operatorname{PDF} f(x)$ and $\operatorname{CDF} F(x)$, the hazard rate function is defined as

$$
h(x)=\lim _{\Delta x \rightarrow 0} \frac{P(X<x+\Delta x \mid X>x)}{\Delta x}=\frac{f(x)}{1-F(x)}
$$

The hazard rate function is an important quantity characterizing life phenomena. For the BEL distribution, the hazard rate function is introduced by the following theorem:

Theorem 3.3. The hazard rate function for the BEL distribution is given by

$$
h(x)=\frac{\alpha \theta^{2}(1+x) e^{-\theta x}\left[1-\left(1-\frac{\theta+1+\theta x}{\theta+1} e^{-\theta x}\right)^{\alpha}\right]^{b-1}\left(1-\frac{\theta+1+\theta x}{\theta+1} e^{-\theta x}\right)^{\alpha a-1}}{(\theta+1)\left[B(a, b)-\alpha \sum_{j, k=0}^{\infty} \sum_{l=0}^{k}\left(\frac{\theta}{\theta+1}\right)^{l} \frac{(-1)^{j+k} \Gamma(b) \Gamma[\alpha(a+j)] x^{l} e^{-\theta k x}}{\Gamma(b-j) \Gamma[\alpha(a+j)+1-k](k-l) ! j ! l !}\right]}
$$

Proof. This can be easily deduced by using (2.5), (3.1) and (3.8).

Figure 2 illustrates some of possible shapes of the hazard rate function of the BEL distribution for selected values of the parameters $a, b, \alpha$ and $\theta$. 


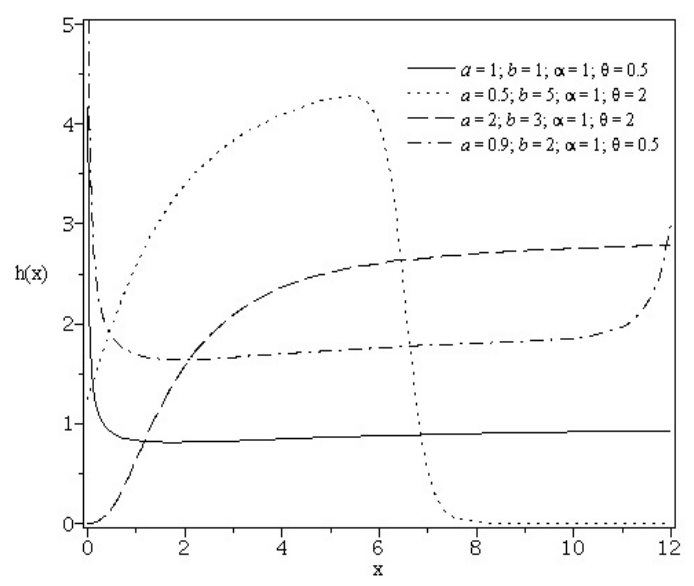

Fig. 2. The hazard rate function of the BEL distribution for different values of the parameters.

\subsection{Moments}

Many of the interesting characteristics and features of a distribution can be studied through its moments (e.g. tendency, dispersion, skewness and kurtosis). Therefore, it is customary to derive the moments when a new distribution is proposed.

Theorem 3.4. The nth moment of BEL distribution, for $b>0$ and $\alpha(a+j)>0$ real non-integers, is given by

$$
\begin{aligned}
E\left(X^{n}\right) & =\frac{\alpha \theta^{2}}{(\theta+1) B(a, b)} \sum_{j, k=0}^{\infty} \sum_{l=0}^{k} \frac{(-1)^{j+k} \Gamma(b) \Gamma[\alpha(a+j)](n+l) !}{\Gamma(b-j) \Gamma[\alpha(a+j)-k](k-l) ! j ! l !}\left(\frac{\theta}{\theta+1}\right)^{l} \\
& \times \frac{[\theta(k+1)+n+l+1]}{[\theta(k+1)]^{n+l+2}}
\end{aligned}
$$

If $b>0$ is an integer, the index $j$ stops at $b-1$. If $\alpha(a+j)>0$ is an integer, the index $k$ stops at $\alpha(a+j)-1$.

Proof. Using the form in (3.4), we can write

$$
\begin{aligned}
E\left(X^{n}\right) & =\int_{0}^{\infty} x^{n} f(x) d x \\
& =\frac{\alpha \theta^{2}}{(\theta+1) B(a, b)} \sum_{j, k=0}^{\infty} \sum_{l=0}^{k} \frac{(-1)^{j+k} \Gamma(b) \Gamma[\alpha(a+j)]}{\Gamma(b-j) \Gamma[\alpha(a+j)-k](k-l) ! j ! l !}\left(\frac{\theta}{\theta+1}\right)^{l} \\
& \times \int_{0}^{\infty} x^{n+l}(1+x) e^{-\theta(k+1) x} d x
\end{aligned}
$$

By setting $t=\theta(k+1) x$ and using the definition of the gamma function (3.2), we obtain

$$
\begin{aligned}
E\left(X^{n}\right) & =\frac{\alpha \theta^{2}}{(\theta+1) B(a, b)} \sum_{j, k=0}^{\infty} \sum_{l=0}^{k} \frac{(-1)^{j+k} \Gamma(b) \Gamma[\alpha(a+j)](n+l) !}{\Gamma(b-j) \Gamma[\alpha(a+j)-k](k-l) ! j ! l !}\left(\frac{\theta}{\theta+1}\right)^{l} \\
& \times \frac{[\theta(k+1)+n+l+1]}{[\theta(k+1)]^{n+l+2}}
\end{aligned}
$$


this completes the proof.

In particular, the mean for the BEL distribution is given by

$$
\begin{aligned}
\mu & =E(X)=\frac{\alpha \theta^{2}}{(\theta+1) B(a, b)} \sum_{j, k=0}^{\infty} \sum_{l=0}^{k} \frac{(-1)^{j+k} \Gamma(b) \Gamma[\alpha(a+j)](l+1)}{\Gamma(b-j) \Gamma[\alpha(a+j)-k](k-l) ! j !}\left(\frac{\theta}{\theta+1}\right)^{l} \\
& \times \frac{[\theta(k+1)+l+2]}{[\theta(k+1)]^{l+3}}
\end{aligned}
$$

\subsection{Moments generating function and the characteristic function}

Here, we derived the moment generating function and the characteristic function of BEL distribution.

Theorem 3.5. The moment generating function of BEL distribution is given by

$$
\begin{aligned}
M(t) & =\frac{\alpha \theta^{2}}{(\theta+1) B(a, b)} \sum_{j, k=0}^{\infty} \sum_{l=0}^{k}\left(\frac{\theta}{\theta+1}\right)^{l} \frac{(-1)^{j+k} \Gamma(b)[\alpha(a+j)]}{\Gamma(b-j) \Gamma[\alpha(a+j)-k](k-l) ! j !} \\
& \times \frac{[\theta(k+1)-t+l+1]}{[\theta(k+1)-t]^{l+1}}
\end{aligned}
$$

where $t<\theta(k+1)$. The corresponding characteristic function is

$$
\begin{aligned}
\phi(t) & =\frac{\alpha \theta^{2}}{(\theta+1) B(a, b)} \sum_{j, k=0}^{\infty} \sum_{l=0}^{k}\left(\frac{\theta}{\theta+1}\right)^{l} \frac{(-1)^{j+k} \Gamma(b)[\alpha(a+j)]}{\Gamma(b-j) \Gamma[\alpha(a+j)-k](k-l) ! j !} \\
& \times \frac{[\theta(k+1)-i t+l+1]}{[\theta(k+1)-i t]^{l+1}}
\end{aligned}
$$

where $i=\sqrt{-1}$.

Proof. Using the form in (3.4), we can write

$$
\begin{aligned}
M(t) & =\int_{0}^{\infty} \exp (t x) g(x) d x \\
& =\frac{\alpha \theta^{2}}{(\theta+1) B(a, b)} \sum_{j, k=0}^{\infty} \sum_{l=0}^{k}\left(\frac{\theta}{\theta+1}\right)^{l} \frac{(-1)^{j+k} \Gamma(b) \Gamma[\alpha(a+j)]}{\Gamma(b-j) \Gamma[\alpha(a+j)-k](k-l) ! j ! l !} \\
& \times \int_{0}^{\infty} x^{l}(1+x) e^{-[\theta(k+1)-t] x} d x
\end{aligned}
$$

By setting $z=[\theta(k+1)-t] x$ with $\theta(k+1)>t$ and using the definition of the gamma function, we obtain

$$
\begin{aligned}
M(t) & =\frac{\alpha \theta^{2}}{(\theta+1) B(a, b)} \sum_{j, k=0}^{\infty} \sum_{l=0}^{k}\left(\frac{\theta}{\theta+1}\right)^{l} \frac{(-1)^{j+k} \Gamma(b)[\alpha(a+j)]}{\Gamma(b-j) \Gamma[\alpha(a+j)-k](k-l) ! j !} \\
& \times \frac{[\theta(k+1)-t+l+1]}{[\theta(k+1)-t]^{l+1}}
\end{aligned}
$$




\section{Characterizations of the model}

Characterizations of distributions are important to many researchers in the applied fields. An investigator will be vitally interested to know if their model fits the requirements of a particular distribution. To this end, one will depend on the characterizations of this distribution which provide conditions under which the underlying distribution is indeed that particular distribution. Various characterizations of distributions have been established in many different directions. In this section, several characterizations of (BEL) distribution are presented. These characterizations are based on: (i) a simple relationship between two truncated moments; (ii) the hazard function of the random variable; (iii) a single function of the random variable.

\subsection{Characterizations based on truncated moments}

In this subsection we present characterizations of (BEL) distribution in terms of a simple relationship between two truncated moments. Our characterization results presented here will employ an interesting result due to Glänzel [8] (Theorem G, below). The advantage of the characterizations given here is that, $c d f F$ need not have a closed form and are given in terms of an integral whose integrand depends on the solution of a first order differential equation, which can serve as a bridge between probability and differential equation.

Theorem G. Let $(\Omega, \mathscr{F}, \mathbf{P})$ be a given probability space and let $H=[a, b]$ be an interval

for some $a<b \quad(a=-\infty, b=\infty$ might as well be allowed). Let $X: \Omega \rightarrow H$ be a continuous random variable with the distribution function $F$ and let $g$ and $h$ be two real functions defined on $H$ such that

$$
\mathbf{E}[g(X) \mid X \geq x]=\mathbf{E}[h(X) \mid X \geq x] \eta(x), \quad x \in H,
$$

is defined with some real function $\eta$. Assume that $g, h \in C^{1}(H), \eta \in C^{2}(H)$ and $F$ is twice continuously differentiable and strictly monotone function on the set $H$. Finally, assume that the equation $h \eta=g$ has no real solution in the interior of $H$. Then $F$ is uniquely determined by the functions $g, h$ and $\eta$, particularly

$$
F(x)=\int_{a}^{x} C\left|\frac{\eta^{\prime}(u)}{\eta(u) h(u)-g(u)}\right| \exp (-s(u)) d u
$$

where the function $s$ is a solution of the differential equation $s^{\prime}=\frac{\eta^{\prime} h}{\eta h-g}$ and $C$ is a constant, chosen to make $\int_{H} d F=1$.

Clearly, Theorem $\mathrm{G}$ can be stated in terms of two functions $g$ and $\eta$ by taking $h(x) \equiv 1$, which will reduce the condition given in Theorem $\mathrm{G}$ to $E[g(X) \mid X \geq x]=\eta(x)$. However, adding an extra function will give a lot more flexibility, as far as its application is concerned. 
Proposition 4.1. Let $X: \Omega \rightarrow(0, \infty)$ be a continuous random variable and let $h(x)=$ $\left[1-\left(1-\frac{\theta+1+\theta x}{\theta+1} e^{-\theta x}\right)^{\alpha}\right]^{1-b}$ and $g(x)=\left(1-\frac{\theta+1+\theta x}{\theta+1} e^{-\theta x}\right)^{\alpha a} h(x)$ for $x \in(0, \infty)$. The $p d f$ of $X$ is (2.5) if and only if the function $\eta$ defined in Theorem $\mathrm{G}$ has the form

$$
\eta(x)=\frac{1}{2}\left\{1+\left(1-\frac{\theta+1+\theta x}{\theta+1} e^{-\theta x}\right)^{\alpha a}\right\}, \quad x>0 .
$$

Proof. Let $X$ have density (2.5), then

$$
(1-F(x)) \mathbf{E}[h(X) \mid X \geq x]=\frac{1}{a B(a, b)}\left\{1-\left(1-\frac{\theta+1+\theta x}{\theta+1} e^{-\theta x}\right)^{\alpha a}\right\}, \quad x>0,
$$

and

$$
(1-F(x)) \mathbf{E}[g(X) \mid X \geq x]=\frac{1}{2 a B(a, b)}\left\{1-\left(1-\frac{\theta+1+\theta x}{\theta+1} e^{-\theta x}\right)^{2 \alpha a}\right\}, \quad x>0,
$$

and finally

$$
\eta(x) h(x)-g(x)=\frac{h(x)}{2}\left\{1-\left(1-\frac{\theta+1+\theta x}{\theta+1} e^{-\theta x}\right)^{\alpha a}\right\}>0 \text { for } x>0 .
$$

Conversely, if $\eta$ is given as above, then

$$
s^{\prime}(x)=\frac{\eta^{\prime}(x) h(x)}{\eta(x) h(x)-g(x)}=\frac{\frac{\alpha a \theta^{2}(1+x)}{\theta+1} e^{-\theta x}\left(1-\frac{\theta+1+\theta x}{\theta+1} e^{-\theta x}\right)^{\alpha a-1}}{\left\{1-\left(1-\frac{\theta+1+\theta x}{\theta+1} e^{-\theta x}\right)^{\alpha a}\right\}}, x>0,
$$

and hence

$$
s(x)=-\ln \left\{1-\left(1-\frac{\theta+1+\theta x}{\theta+1} e^{-\theta x}\right)^{\alpha a}\right\}, \quad x>0 .
$$

Now, in view of Theorem G, $X$ has density (2.5).

Corollary 4.1. Let $X: \Omega \rightarrow(0, \infty)$ be a continuous random variable and let $h(x)$ be as in Proposition 4.1. The $p d f$ of $X$ is (2.5) if and only if there exist functions $g$ and $\eta$ defined in Theorem $\mathrm{G}$ satisfying the differential equation 


$$
\frac{\eta^{\prime}(x) h(x)}{\eta(x) h(x)-g(x)}=\frac{\alpha a \theta^{2}(1+x) e^{-\theta x}\left(1-\frac{\theta+1+\theta x}{\theta+1} e^{-\theta x}\right)^{\alpha a-1}}{(\theta+1)\left\{1-\left(1-\frac{\theta+1+\theta x}{\theta+1} e^{-\theta x}\right)^{\alpha a}\right\}}, \quad x>0 .
$$

Remarks 4.1. (a) The general solution of the differential equation in Corollary 4.1 is

$$
\begin{aligned}
\eta(x)= & {\left[1-\left(1-\frac{\theta+1+\theta x}{\theta+1} e^{-\theta x}\right)^{\alpha}\right]^{-1} \times } \\
& {\left[\begin{array}{c}
-\int \frac{\alpha a \theta^{2}(1+x)}{\theta+1} e^{-\theta x}\left(1-\frac{\theta+1+\theta x}{\theta+1} e^{-\theta x}\right)^{\alpha a-1} \times \\
{\left[1-\left(1-\frac{\theta+1+\theta x}{\theta+1} e^{-\theta x}\right)^{\alpha}\right]^{b-1} g(x) d x+D}
\end{array}\right], }
\end{aligned}
$$

for $x>0$, where $D$ is a constant. One set of appropriate functions is given in Proposition 4.1 with $D=\frac{1}{2}$

(b) Clearly there are other triplets of functions $(h, g, \eta)$ satisfying the conditions of Theorem G. We presented one such triplet in Proposition 4.1.

\subsection{Characterization based on hazard function}

The hazard function of twice differentiable distribution function, $F$, satisfies the first order differential equation

$$
\frac{h_{F}^{\prime}(x)}{h_{F}(x)}-h_{F}(x)=q(x)
$$

where $q(x)$ is an appropriate integrable function. Although this differential equation has an obvious form since

$$
\frac{h_{F}^{\prime}(x)}{h_{F}(x)}-h_{F}(x)=\frac{f^{\prime}(x)}{f(x)}
$$

for many univariate continuous distributions (4.1) seems to be the only differential equation in

terms of the hazard function. The goal of the characterization based on hazard function is to establish a differential equation in terms of hazard function, which has as simple form as possible and is not of the trivial form (4.1) . For some general families of distributions, like (BEL) family, this may not be possible, but possible for their sub-families. Here, we present a characterization of the sub-model (2) (which includes sub-model (5)) of (BEL) model based on a nontrivial differential equation in terms of the hazard function.

Proposition 4.2. Let $X: \Omega \rightarrow(0, \infty)$ be a continuous random variable. The $p d f$ of $X$ is 
(2.7), for $\alpha c=1$, if and only if its hazard function $h_{F}(x)$ satisfies the differential equation

$$
h_{F}^{\prime}(x)+\theta(\theta+1+\theta x)^{-1} h_{F}(x)=\theta^{2}(\theta+1+\theta x)^{-1}, 0<x<\infty
$$

with initial condition $h_{F}(0)=\theta^{2}(\theta+1)$.

Proof: If $X$ has $p d f(2.7)$ with $\alpha c=1$, then clearly (4.2) holds. Now, if (4.2) holds, then

$$
\frac{d}{d x}\left\{(\theta+1+\theta x) h_{F}(x)\right\}=\theta^{2} x
$$

from which we have

$$
(\theta+1+\theta x) h_{F}(x)=\theta^{2} x+\theta^{2}
$$

or

$$
h_{F}(x)=\frac{f(x)}{1-F(x)}=\theta^{2}(1+x)(\theta+1+\theta x)^{-1}
$$

Integrating both sides of (4.3) from 0 to $x$, we arrive at

$$
-\ln (1-F(x))=-\ln (\theta+1+\theta x)+\ln (\theta+1)-\theta x .
$$

from which we obtain

$$
F(x)=1-\frac{(\theta+1+\theta x) e^{-\theta x}}{(\theta+1)}, \quad x \geq 0 .
$$

\subsection{Characterizations based on single function of the random variable}

In this subsection we employ a single function $\psi$ of $X$ and state characterization results in terms of $\psi(X)$. The following propositions have already appeared a Technical Report ( see [10]), so we will just state them here for the sake of completeness.

Proposition 4.3. Let $X: \Omega \rightarrow(a, b)$ be a continuous random variable with $c d f F$. Let $\psi(x)$ be a differentiable function on $(a, b)$ with $\lim _{x \rightarrow a} \psi(x)=1$. Then for $\delta \neq 1$,

$$
E[\psi(X) \mid X>x]=\delta \psi(x), \quad x \in(a, b),
$$


if and only if

$$
\psi(x)=(1-F(x))^{\frac{1}{\delta}-1}, \quad x \in(a, b)
$$

Proposition 4.4. Let $X: \Omega \rightarrow(a, b)$ be a continuous random variable with $c d f F$. Let $\psi_{1}(x)$ be a differentiable function on $(a, b)$ with $\lim _{x \rightarrow b} \psi_{1}(x)=1$. Then for $\delta_{1} \neq 1$,

$$
E\left[\psi_{1}(X) \mid X<x\right]=\delta_{1} \psi_{1}(x), \quad x \in(a, b)
$$

if and only if

$$
\psi_{1}(x)=(F(x))^{\frac{1}{\delta_{1}}-1}, \quad x \in(a, b)
$$

Remark 4.1. All of the sub-models (1)-(5) can be characterized via the above two Propositions with appropriate functions $\psi(x)$ or $\psi_{1}(x)$.

\section{Inference}

In this section, we consider estimation of the three parameters by the methods of moments and maximum likelihood. Suppose $x_{1}, \ldots, x_{n}$ is a random sample of size $n$ from the BEL distribution given by (2.5). Under the method of moments, equating $E\left(X^{r}\right)$ with the corresponding sample moment,

$$
M_{r}=\frac{1}{n} \sum_{i=1}^{n} x_{i}^{r}, r=1,2,3,4
$$

respectively, one obtains the system of equations

$$
\begin{aligned}
M_{r} & =\frac{\alpha \theta^{2}}{(\theta+1) B(a, b)} \sum_{j, k=0}^{\infty} \sum_{l=0}^{k} \frac{(-1)^{j+k} \Gamma(b) \Gamma[\alpha(a+j)](r+l) !}{\Gamma(b-j) \Gamma[\alpha(a+j)-k](k-l) ! j ! l !}\left(\frac{\theta}{\theta+1}\right)^{l} \\
& \times \frac{[\theta(k+1)+r+l+1]}{[\theta(k+1)]^{r+l+2}}
\end{aligned}
$$

which can be solved simultaneously to give estimates for $a, b, \alpha$ and $\theta$.

Now consider estimation by the method of maximum likelihood. The log-likelihood for a random sample $x_{1}, \ldots, x_{n}$ from the BEL distribution given by (2.5) is 


$$
\begin{aligned}
\log L(a, b, \alpha, \theta) & =n \log \alpha+2 n \log \theta-n \log (\theta+1)+n[\log \Gamma(a+b)-\log \Gamma(a)-\log \Gamma(b)] \\
& -\theta \sum_{i=1}^{n} x_{i}+\sum_{i=1}^{n} \log \left(1+x_{i}\right)+(\alpha a-1) \sum_{i=1}^{n} \log \left(1-\frac{\theta+1+\theta x_{i}}{\theta+1} e^{-\theta x_{i}}\right) \\
& +(b-1) \sum_{i=1}^{n} \log \left[1-\left(1-\frac{\theta+1+\theta x_{i}}{\theta+1} e^{-\theta x_{i}}\right)^{\alpha}\right]
\end{aligned}
$$

Differentiating the log-likelihood with respect $a, b, \alpha$ and $\theta$, respectively, and setting the result equal to zero, we have

$$
\begin{aligned}
& \frac{\partial \log L}{\partial a}=n[\psi(a+b)-\psi(a)]+\alpha \sum_{i=1}^{n} \log \left(1-\frac{\theta+1+\theta x_{i}}{\theta+1} e^{-\theta x_{i}}\right)=0 \\
& \frac{\partial \log L}{\partial b}=n[\psi(a+b)-\psi(b)]+\sum_{i=1}^{n} \log \left[1-\left(1-\frac{\theta+1+\theta x_{i}}{\theta+1} e^{-\theta x_{i}}\right)^{\alpha}\right]=0 \\
& \frac{\partial \log L}{\partial \alpha}=\frac{n}{\alpha}-\sum_{i=1}^{n} \frac{(b-1)\left[\theta+1-\left(\theta+1+\theta x_{i}\right) e^{-\theta x}\right]^{\alpha}}{(\theta+1)^{\alpha}-\left[\theta+1-\left(\theta+1+\theta x_{i}\right) e^{-\theta x}\right]^{\alpha}} \log \left(1-\frac{\theta+1+\theta x_{i}}{\theta+1} e^{-\theta x_{i}}\right) \\
& +a \sum_{i=1}^{n} \log \left(1-\frac{\theta+1+\theta x_{i}}{\theta+1} e^{-\theta x_{i}}\right)=0 \\
& \frac{\partial \log L}{\partial \theta}=\frac{2 n}{\theta}-\frac{n}{\theta+1}-\sum_{i=1}^{n} x_{i}+(\alpha a-1) \sum_{i=1}^{n} \frac{\theta x_{i}\left[(\theta+1)\left(1+x_{i}\right)+1\right] e^{-\theta x_{i}}}{(\theta+1)\left[\theta+1-\left(\theta+1+\theta x_{i}\right) e^{-\theta x_{i}}\right]} \\
& -\alpha(b-1) \sum_{i=1}^{n} \frac{\theta x_{i}\left[\theta+1-\left(\theta+1+\theta x_{i}\right) e^{-\theta x_{i}}\right]^{\alpha-1}\left[(\theta+1)\left(1+x_{i}\right)+1\right] e^{-\theta x_{i}}}{(\theta+1)\left\{(\theta+1)^{\alpha}\left[\theta+1-\left(\theta+1+\theta x_{i}\right) e^{-\theta x_{i}}\right]^{\alpha}\right\}}=0
\end{aligned}
$$

where $\psi(x)=d \log \Gamma(x) / d x$ is the digamma function.

\section{Application}

In this section, we use the lifetime data set given by Table 1 to compare the fit of the BEL distribution with four distributions: beta Lindley, gamma, Lindley and Weibull.

The data set given in Table 1 represents the relief times of twenty patients receiving an analgesic. This data set was taken from [9]. We would like to emphasize that the aim here is not to provide a complete statistical modeling or inferences for the data set involved. 
Table 1. Relief times of twenty patients.

\begin{tabular}{llllllllll}
\hline 1.1 & 1.4 & 1.3 & 1.7 & 1.9 & 1.8 & 1.6 & 2.2 & 1.7 & 2.7 \\
4.1 & 1.8 & 1.5 & 1.2 & 1.4 & 3.0 & 1.7 & 2.3 & 1.6 & 2.0 \\
\hline
\end{tabular}

The maximum likelihood estimates and the Akaike Information Criterion (AIC) values for the fitted distributions are reported in Table 2. The results show that the BEL distribution provides a significantly better fit than the other four models.

Table 2. The maximum likelihood estimates and AIC of the models based on data set.

\begin{tabular}{llc}
\hline Distribution & \multicolumn{1}{c}{ Maximum Likelihood Estimates } & AIC \\
\hline BEL & $a=10.401, b=0.322, \alpha=39.581, \theta=5.353$ & 38.937 \\
BL & $a=9.300, b=1.717, \theta=1.489$ & 40.932 \\
Gamma & $a=9.670, b=5.089$ & 39.637 \\
Lindley & $\theta=0.816$ & 62.499 \\
Weibull & $a=2.130, b=2.787$ & 45.173 \\
\hline
\end{tabular}

The fitted distributions superimposed to the histogram of the data in Figure 3 reinforce the results found for the BEL model. Figure 4 display the probability plots and supports the results shown in Table 2.

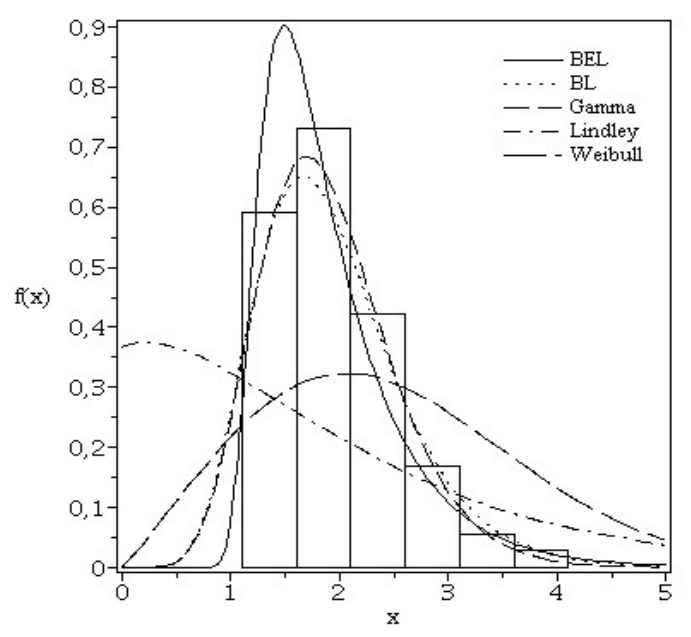

Fig. 3. Histogram and estimated densities. 

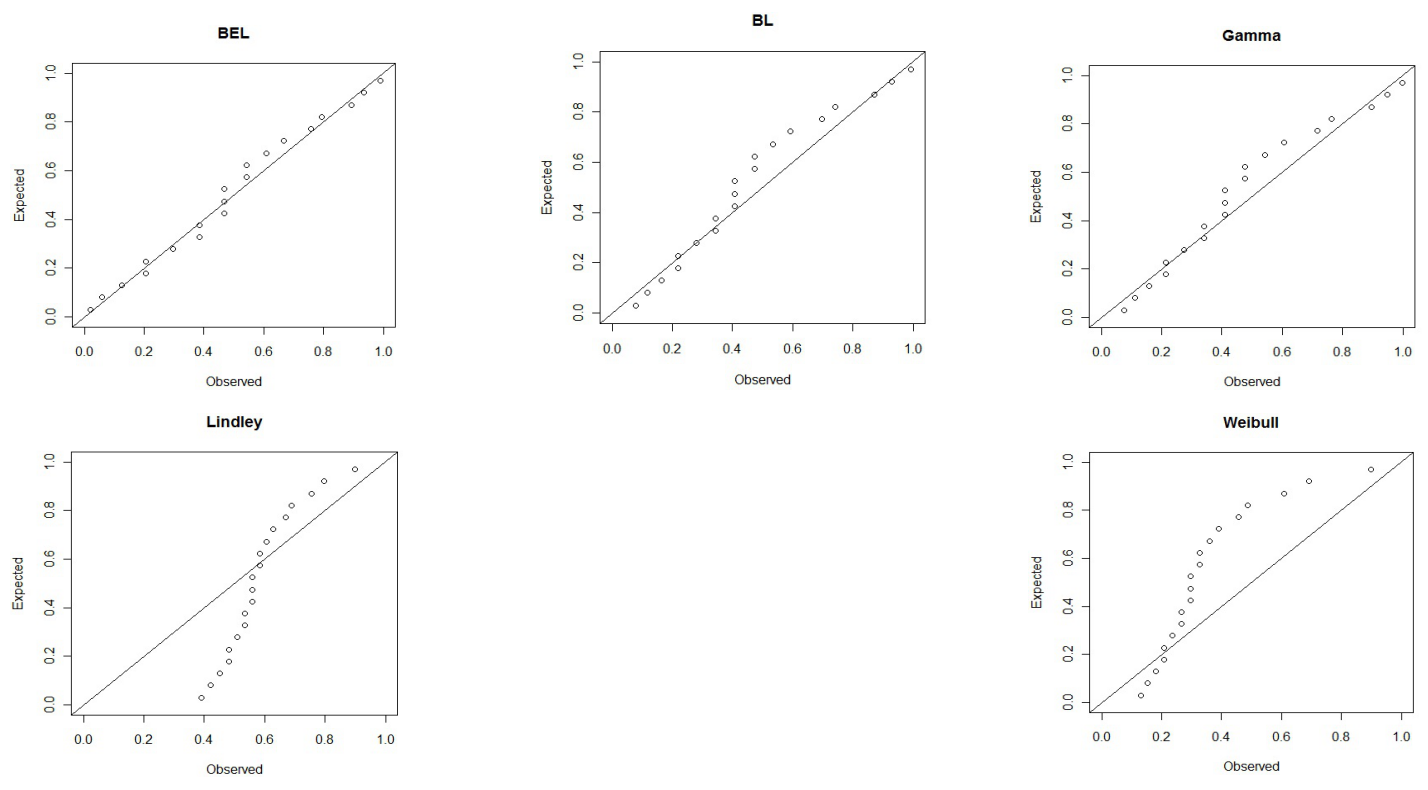

Fig. 4. Probability plots from the fitted BEL, BL, gamma, Lidley and Weibull distributions.

\section{Conclusion}

We proposed a new distribution, named the beta exponentiated Lindley distribution which extends the Lindley distribution. Several properties of the new distribution were investigated, including moments and the failure rate function. Characterizations of the beta exponentiated Lindley distribution are presented. The estimation of parameters by the method of moments and the maximum likelihood have been discussed. An application of the beta exponentiated Lindley distribution to real data show that the new distribution can be used quite effectively to provide better fits than the beta Lindley, gamma, Lindley and Weibull distributions.

\section{References}

[1] A. Akinsete, F. Famoye, and C. Lee, The beta-Pareto distribution, Statistics. 42(6) (2008), pp. 547-563.

[2] A. Alzaatreh, F. Famoye, and C. Lee, Gamma-Pareto distribution and its application, Journal of Modern Applied Statistical Methods. 11(1) (2012), pp. 78-94.

[3] A. Alzaatreh, F. Famoye, and C. Lee, Weibull-Pareto distribution and its applications, Communication in Statistics - Theory and Methods. 42(9) (2013), pp. 1673-1691.

[4] A.N. Balakrishnan, and H.N. Nagaraja, A first course in order statistics, Wiley-Interscience, New York, 1992.

[5] F. Condino and F. Domma, The Beta-Dagum distribution, Communication in Statistics - Theory and Methods. 42(22) (2013), pp. 4070-4090.

[6] I. Elbatal, F. Merovci, and M. Elgarhy, A new generalized Lindley distribution, Mathematical Theory and Modeling. 3(13) (2013), pp. 30-47.

[7] N. Eugene, C. Lee, and F. Famoye, Beta-normal distribution and its applications, Communication in Statistics - Theory and Methods. 4(31) (2002), pp. 497-512.

[8] W. Glänzel, A characterization theorem based on truncated moments and its application to some distribution families, Mathematical Statistics and Probability Theory (Bad Tatzmannsdorf, 1986), Vol. B, Reidel, Dordrecht, 1987, pp. 75-84. 
[9] A.J. Gross and V.A. Clark, Survival distributions: Reliability applications in the biomedical sciences, John Wileyand Sons, New York, 1975.

[10] G.G. Hamedani, On certain generalized gamma convolution distributions II, Technical Report, No. 484, MSCS, Marquette University (2013).

[11] D.V. Lindley, Fiducial distributions and Bayes' theorem, Journal of the Royal Statistical Society B. 20 (1958), pp. 102-107.

[12] C. Lee, F. Famoye, and O. Olumolade, Beta-Weibull distribution, Journal of Modern Applied Statistical Methods. 6(1) (2007), pp. 173-186.

[13] F. Merovci and V.K. Sharma, The beta Lindley distribution: Properties and Applications, Journal of Applied Mathematics. (2014), ID 198951, pp. 1-10.

[14] S. Nadarajah, H.S. Bakouch, and R. Tahmasbi, A generalized Lindley distribution, Sankhya B. 73 (2011), pp. 331-359.

[15] P.F. Paranaba, E.M.M. Ortega, G.M. Cordeiro, and R.R. Pescim, The beta Burr XII distribution with application to lifetime data, Communication in Statistics - Theory and Methods. 55(2) (2011), pp. 1118-1136.

[16] M.A.R. Pascoa, E.M.M. Ortega, and G.M. Cordeiro, The Kumaraswamy generalized gamma distribution with application in survival analysis, Communication in Statistics - Theory and Methods. 8(5) (2011), pp. 411-433.

[17] L.G.B. Pinho, G.M. Cordeiro, and J.S. Nobre, The gamma-exponentiated Weibull distribution, Journal of Statistical Theory and Applications. 11(4) (2012), pp. 379-395.

[18] G.O. Silva, E.M.M. Ortega, and G.M. Cordeiro, The beta modified Weibull distribution, Mathematic and Statistic. 16(3) (2010), pp. 409-430.

[19] H. Zamini and N. Ismail, Negative Binomial-Lindley distribution and its application, Journal of Mathematics and Statistics. 6(1) (2010), pp. 4-9.

[20] R. Shanker, S. Sharma, and R. Shanker, A two-parameter Lindley distribution for modeling waiting and survival times data, Applied Mathematics. 4 (2013), pp. 363-368. 